PROCEEDINGS OF THE

AMERICAN MATHEMATICAL SOCIETY

Volume 137, Number 8, August 2009, Pages 2755-2759

S 0002-9939(09)09866-9

Article electronically published on March 18, 2009

\title{
ON THE COMPLETENESS OF GRADIENT RICCI SOLITONS
}

\author{
ZHU-HONG ZHANG
}

(Communicated by Richard A. Wentworth)

\begin{abstract}
A gradient Ricci soliton is a triple $(M, g, f)$ satisfying $R_{i j}+\nabla_{i} \nabla_{j} f$ $=\lambda g_{i j}$ for some real number $\lambda$. In this paper, we will show that the completeness of the metric $g$ implies that of the vector field $\nabla f$.
\end{abstract}

\section{INTRODUCTION}

Definition 1.1. Let $(M, g, X)$ be a smooth Riemannian manifold with $X$ a smooth vector field. We call $M$ a Ricci soliton if $R i c+\frac{1}{2} \mathcal{L}_{X} g=\lambda g$ for some real number $\lambda$. It is called shrinking when $\lambda>0$, steady when $\lambda=0$, and expanding when $\lambda<0$. If $(M, g, f)$ is a smooth Riemannian manifold, where $f$ is a smooth function such that $(M, g, \nabla f)$ is a Ricci soliton, i.e. $R_{i j}+\nabla_{i} \nabla_{j} f=\lambda g_{i j}$, we call $(M, g, f)$ a gradient Ricci soliton and $f$ the soliton function.

On the other hand, one has the following definition (see chapter 2 of [3]).

Definition 1.2. Let $(M, g(t), X)$ be a smooth Riemannian manifold with a solution $g(t)$ of the Ricci flow on a time interval $(a, b)$ containing 0 , where $X$ is a smooth vector field. We call $(M, g(t), X)$ a self-similar solution if there exist scalars $\sigma(t)$ such that $g(t)=\sigma(t) \varphi_{t}^{*}\left(g_{0}\right)$, where the diffeomorphism $\varphi_{t}$ is generated by $X$. If the vector field $X$ comes from a gradient of a smooth function $f$, then we call $(M, g(t), f)$ a gradient self-similar solution.

It is easy to see that if $(M, g(t), f)$ is a complete gradient self-similar solution, then $(M, g(0), f)$ must be a complete gradient Ricci soliton. Conversely, when $(M, g, f)$ is a complete gradient Ricci soliton and, in addition, the vector field $\nabla f$ is complete, it is well known (see for example Theorem 4.1 of 2]) that there is a complete gradient self-similar solution $(M, g(t), f), t \in(a, b)$ (with $0 \in(a, b)$ ), such that $g(0)=g$. Here we say that a vector field $\nabla f$ is complete if it generates a family of diffeomorphisms $\varphi_{t}$ of $M$ for $t \in(a, b)$.

So when the vector field is complete, the definitions of gradient Ricci soliton and gradient self-similar solution are equivalent. In the literature, people sometimes confuse the gradient Ricci solitons with the gradient self-similar solutions. Indeed, if the gradient Ricci soliton has bounded curvature, then it is not hard to see that the vector field $\nabla f$ is complete. But, in general, the soliton does not have bounded curvature.

Received by the editors September 22, 2008, and, in revised form, December 30, 2008. 2000 Mathematics Subject Classification. Primary 53C20.

Key words and phrases. Completeness, gradient Ricci soliton, gradient self-similar solution.

The author was supported in part by NSFC 10831008 and NKBRPC 2006CB805905.

(C)2009 American Mathematical Society Reverts to public domain 28 years from publication 
The purpose of this paper is to show that the completeness of the metric $g$ of a gradient Ricci soliton $(M, g, f)$ implies that of the vector field $\nabla f$, even though the soliton does not have bounded curvature. Our main result is the following.

Theorem 1.3. Let $(M, g, f)$ be a gradient Ricci soliton. Suppose the metric $g$ is complete. Then we have:

(i) $\nabla f$ is complete;

(ii) $R \geq 0$ if the soliton is steady or shrinking;

(iii) $\exists C \geq 0$, such that $R \geq-C$ if the soliton is expanding.

Indeed, we will show that the vector field $\nabla f$ grows at most linearly and so it is integrable. Hence Definitions 1.1 and 1.2 are equivalent when the metric is complete.

\section{Gradient Ricci solitons}

Let $(M, g, f)$ be a gradient Ricci soliton, i.e., $R_{i j}+\nabla_{i} \nabla_{j} f=\lambda g_{i j}$. By using the contracted second Bianchi identity we get the equation $R+|\nabla f|^{2}-2 \lambda f=$ const.

Definition 2.1. Let $(M, g, f)$ be a gradient shrinking or expanding soliton. By rescaling $g$ and changing $f$ by a constant we can assume $\lambda \in\left\{-\frac{1}{2}, \frac{1}{2}\right\}$ and $R+$ $|\nabla f|^{2}-2 \lambda f=0$. We call such a soliton normalized, and we call $f$ a normalized soliton function.

Proposition 2.2. Let $(M, g, f)$ be a gradient Ricci soliton. Fix $p$ on $M$ and define $d(x) \triangleq d(p, x)$. Then the following hold:

(i) $\triangle R=\langle\nabla f, \nabla R\rangle+2 \lambda R-|R i c|^{2}$.

(ii) Suppose Ric $\leq(n-1) K$ on $B_{r_{0}}(p)$, for some positive numbers $r_{0}$ and $K$. Then for an arbitrary point $x$, outside $B_{r_{0}}(p)$, we have

$$
\triangle d-\langle\nabla f, \nabla d\rangle \leq-\lambda d(x)+(n-1)\left\{\frac{2}{3} K r_{0}+r_{0}^{-1}\right\}+|\nabla f|(p) .
$$

Proof. (i) By using the soliton equation and the contracted second Bianchi identity $\nabla_{i} R=2 g^{j k} \nabla_{j} R_{i k}$, we have

$$
\begin{aligned}
\triangle R & =g^{i j} \nabla_{i} \nabla_{j} R=g^{i j} \nabla_{i}\left(2 g^{k l} R_{j k} \nabla_{l} f\right)=2 g^{i j} g^{k l} \nabla_{i}\left(R_{j k} \nabla_{l} f\right) \\
& =2 g^{i j} g^{k l} \nabla_{i}\left(R_{j k}\right) \nabla_{l} f+2 g^{i j} g^{k l} R_{j k} \nabla_{i} \nabla_{l} f \\
& =g^{k l} \nabla_{k} R \nabla_{l} f+2 g^{i j} g^{k l} R_{j k}\left(\lambda g_{i l}-R_{i l}\right) \\
& =\langle\nabla f, \nabla R\rangle+2 \lambda R-2|R i c|^{2} .
\end{aligned}
$$

(ii) Let $\gamma:[0, d(x)] \rightarrow M$ be a shortest normal geodesic from $p$ to $x$. We may assume that $x$ and $p$ are not conjugate to each other; otherwise, we can understand the differential inequality in the barrier sense. Let $\left\{\dot{\gamma}(0), e_{1}, \cdots, e_{n-1}\right\}$ be an orthonormal basis of $T_{p} M$. Extend this basis parallel along $\gamma$ to form a parallel orthonormal basis $\left\{\dot{\gamma}(t), e_{1}(t), \cdots, e_{n-1}(t)\right\}$ along $\gamma$.

Let $X_{i}(t), i=1,2, \cdots, n-1$, be the Jacobian fields along $\gamma$ with $X_{i}(0)=0$ and $X_{i}(d(x))=e_{i}(d(x))$. Then it is well-known that (see for example [4])

$$
\triangle d(x)=\sum_{i=1}^{n-1} \int_{0}^{d(x)}\left[\left|\dot{X}_{i}\right|^{2}-R\left(\dot{\gamma}, X_{i}, \dot{\gamma}, X_{i}\right)\right] d t .
$$


Define vector fields $Y_{i}, i=1,2, \cdots, n-1$, along $\gamma$ as follows:

$$
Y_{i}(t)= \begin{cases}\frac{t}{r_{0}} e_{i}(t), & \text { if } t \in\left[0, r_{0}\right] \\ e_{i}(t), & \text { if } t \in\left[r_{0}, d(x)\right] .\end{cases}
$$

Then by using the standard index comparison theorem we have

$$
\begin{aligned}
\triangle d(x) & =\sum_{i=1}^{n-1} \int_{0}^{d(x)}\left[\left|\dot{X}_{i}\right|^{2}-R\left(\dot{\gamma}, X_{i}, \dot{\gamma}, X_{i}\right)\right] d t \\
& \leq \sum_{i=1}^{n-1} \int_{0}^{d(x)}\left[\left|\dot{Y}_{i}\right|^{2}-R\left(\dot{\gamma}, Y_{i}, \dot{\gamma}, Y_{i}\right)\right] d t \\
& =\int_{0}^{r_{0}}\left[\frac{n-1}{r_{0}^{2}}-\frac{t^{2}}{r_{0}^{2}} \operatorname{Ric}(\dot{\gamma}, \dot{\gamma})\right] d t+\int_{r_{0}}^{d(x)}[-\operatorname{Ric}(\dot{\gamma}, \dot{\gamma}] d t \\
& =-\int_{0}^{d(x)} \operatorname{Ric}(\dot{\gamma}, \dot{\gamma}) d t+\int_{0}^{r_{0}}\left[\frac{n-1}{r_{0}^{2}}+\left(1-\frac{t^{2}}{r_{0}^{2}}\right) \operatorname{Ric}(\dot{\gamma}, \dot{\gamma})\right] d t \\
& \leq-\int_{\gamma} \operatorname{Ric}(\dot{\gamma}, \dot{\gamma}) d t+(n-1)\left\{\frac{2}{3} K r_{0}+r_{0}^{-1}\right\}
\end{aligned}
$$

On the other hand,

$\langle\nabla f, \nabla d\rangle(x)=\nabla_{\dot{\gamma}} f(x)=\int_{0}^{d(x)}\left(\frac{d}{d t} \nabla_{\dot{\gamma}} f\right) d t+\nabla_{\dot{\gamma}} f(p) \geq \int_{\gamma}\left(\nabla_{\dot{\gamma}} \nabla_{\dot{\gamma}} f\right) d t-|\nabla f|(p)$.

Using the soliton equation, we have

$$
\begin{aligned}
\triangle d-\langle\nabla f, \nabla d\rangle & \leq-\int_{\gamma}\left[\operatorname{Ric}(\dot{\gamma}, \dot{\gamma})+\nabla_{\dot{\gamma}} \nabla_{\dot{\gamma}} f\right] d t+(n-1)\left\{\frac{2}{3} K r_{0}+r_{0}^{-1}\right\}+|\nabla f|(p) \\
& =-\lambda d(x)+(n-1)\left\{\frac{2}{3} K r_{0}+r_{0}^{-1}\right\}+|\nabla f|(p) .
\end{aligned}
$$

Now we are ready to prove Theorem 1.3.

Proof. Fix a point $p$ on $M$, and define $d(x) \triangleq d(p, x)$. We divide the argument into three steps.

Step 1. We want to prove a curvature estimate in the following assertion.

Claim. For any gradient Ricci soliton, we have:

(i) If the soliton is shrinking or steady, then $R \geq 0$.

(ii) If the soliton is expanding, then there exists a nonnegative constant $C=C(n)$ such that $R \geq \lambda C$.

We only prove the case (i), $\lambda \geq 0$. Note that there is a positive constant $r_{0}$, such that Ric $\leq(n-1) r_{0}^{-2}$ on $B_{r_{0}}(p)$, and $|\nabla f|(p) \leq(n-1) r_{0}^{-1}$. Then by Proposition 2.2, we have

for any $x \notin B_{r_{0}}(p)$.

$$
\triangle d-\langle\nabla f, \nabla d\rangle \leq \frac{8}{3}(n-1) r_{0}^{-1}
$$

For any fixed constant $A>2$, we consider the function $u(x)=\varphi\left(\frac{d(x)}{A r_{0}}\right) R(x)$, where $\varphi$ is a fixed smooth nonnegative decreasing function such that $\varphi=1$ on $\left(-\infty, \frac{1}{2}\right]$, and $\varphi=0$ on $[1, \infty)$. 
Then by Proposition 2.2, we have

$$
\begin{aligned}
\triangle u & =R \triangle \varphi+\varphi \triangle R+2\langle\nabla \varphi, \nabla R\rangle \\
& =R\left(\varphi^{\prime \prime} \frac{1}{\left(A r_{0}\right)^{2}}+\varphi^{\prime} \frac{1}{A r_{0}} \triangle d\right)+\varphi\left(\langle\nabla f, \nabla R\rangle+2 \lambda R-|R i c|^{2}\right)+2\langle\nabla \varphi, \nabla R\rangle .
\end{aligned}
$$

If $\min _{x \in M} u \geq 0$, then $R \geq 0$ on $B_{\frac{1}{2} A r_{0}}(p)$. Otherwise, $\min _{x \in M} u<0$. Then there exists some point $x_{1} \in B_{A r_{0}}(p)$ such that $u\left(x_{1}\right)=\varphi R\left(x_{1}\right)=\min _{x \in M} u<0$. Because $u\left(x_{1}\right)$ is the minimum of the function $u(x)$, we have $\varphi^{\prime} R\left(x_{1}\right)>0, \nabla u\left(x_{1}\right)=0$, and $\triangle u\left(x_{1}\right) \geq 0$.

Let us first consider the case that $x_{1} \notin B_{r_{0}}(p)$. Then by direct computation, we have

$$
\begin{aligned}
\triangle u\left(x_{1}\right)= & \left(\frac{\varphi^{\prime \prime}}{\varphi} \frac{1}{\left(A r_{0}\right)^{2}}+\frac{\varphi^{\prime}}{\varphi} \frac{1}{A r_{0}} \triangle d\right) u\left(x_{1}\right)-\frac{\varphi^{\prime}}{\varphi} \frac{1}{A r_{0}}\langle\nabla f, \nabla d\rangle u\left(x_{1}\right) \\
& +2 \lambda u\left(x_{1}\right)-\varphi|R i c|^{2}-\frac{\varphi^{\prime 2}}{\varphi^{2}} \frac{2}{\left(A r_{0}\right)^{2}} u\left(x_{1}\right) \\
\leq & \left(\frac{\varphi^{\prime \prime}}{\varphi} \frac{1}{\left(A r_{0}\right)^{2}}-\frac{\varphi^{\prime 2}}{\varphi^{2}} \frac{2}{\left(A r_{0}\right)^{2}}\right) u\left(x_{1}\right)-\frac{2}{n} \varphi R^{2} \\
& +\frac{\varphi^{\prime}}{\varphi} \frac{1}{A r_{0}} u\left(x_{1}\right)(\triangle d-\langle\nabla f, \nabla d\rangle) . \\
\leq & \left(\frac{\varphi^{\prime \prime}}{\varphi} \frac{1}{\left(A r_{0}\right)^{2}}-\frac{\varphi^{\prime 2}}{\varphi^{2}} \frac{2}{\left(A r_{0}\right)^{2}}\right) u\left(x_{1}\right)-\frac{2}{n} \frac{1}{\varphi} u\left(x_{1}\right)^{2} \\
& +\frac{8}{3}(n-1) \frac{\varphi^{\prime}}{\varphi} \frac{1}{A r_{0}^{2}} u\left(x_{1}\right) \\
= & \frac{u\left(x_{1}\right)}{\varphi}\left\{\left(\varphi^{\prime \prime} \frac{1}{\left(A r_{0}\right)^{2}}-\frac{\varphi^{\prime 2}}{\varphi} \frac{2}{\left(A r_{0}\right)^{2}}\right)+\frac{8}{3}(n-1) \varphi^{\prime} \frac{1}{A r_{0}^{2}}-\frac{2}{n} u\left(x_{1}\right)\right\} \\
\leq & \frac{\left|u\left(x_{1}\right)\right|}{\varphi}\left\{\frac{\varphi^{\prime 2}}{\varphi} \frac{2}{A r_{0}^{2}}+\frac{8(n-1)}{3}\left(-\varphi^{\prime}\right) \frac{1}{A r_{0}^{2}}+\left|\varphi^{\prime \prime}\right| \frac{1}{A r_{0}^{2}}-\frac{2}{n}\left|u\left(x_{1}\right)\right|\right\} .
\end{aligned}
$$

Note that there exists a constant $\widetilde{C}=\widetilde{C}(\varphi)$ such that $\left|\varphi^{\prime}\right| \leq \widetilde{C}, \frac{\varphi^{\prime 2}}{\varphi} \leq \widetilde{C}$, and $\left|\varphi^{\prime \prime}\right| \leq \widetilde{C}$. So

$$
\left|u\left(x_{1}\right)\right| \leq \frac{C}{A r_{0}^{2}},
$$

where the constant $C=C(\varphi, n)$, i.e., $R \geq-\frac{C}{A r_{0}^{2}}$ on $B_{\frac{1}{2}} A r_{0}(p)$.

We now consider the remaining case that $x_{1} \in B_{r_{0}}(p)$. Then $\varphi^{\prime}\left(x_{1}\right)=\varphi^{\prime \prime}\left(x_{1}\right)=$ 0 , and we have

$$
\triangle u\left(x_{1}\right)=2 \lambda u\left(x_{1}\right)-\varphi|R i c|^{2} \leq\left|u\left(x_{1}\right)\right|\left[-2 \lambda-\frac{2}{n}\left|u\left(x_{1}\right)\right|\right] .
$$

Since $\lambda \geq 0$, we have $\left|u\left(x_{1}\right)\right| \leq 0$, i.e., $u\left(x_{1}\right)=0$. This is a contradiction.

Combining the above two cases, we have $R \geq-\frac{C}{A r_{0}^{2}}$ on $B_{\frac{1}{2} A r_{0}}(p)$ for any $A>2$, which implies that $R \geq 0$ on $M$.

The proof of (ii) is similar.

Step 2. We next want to show that the gradient field grows at most linearly.

Claim. For any gradient Ricci soliton, there exist constants $a$ and $b$ depending only on the soliton, such that

(i) $|\nabla f|(x) \leq|\lambda| d(x)+a$; 
(ii) $|f|(x) \leq \frac{|\lambda|}{2} d(x)^{2}+a d(x)+b$.

For any point $x$ on $M$, we connect $p$ and $x$ by a shortest normal geodesic $\gamma(t), t \in$ $[0, d(x)]$.

We first consider that the soliton is steady. Then $R \geq 0$ and $R+|\nabla f|^{2}=C \geq 0$, so we have $|\nabla f| \leq \sqrt{C}$.

Secondly, we consider that the soliton is shrinking. Without loss of generality, we may assume the soliton is normalized. So $R \geq 0$ and $R+|\nabla f|^{2}-f=0$; these imply $f \geq|\nabla f|^{2}$. Let $h(t)=f(\gamma(t))$. Then

$$
\left|h^{\prime}\right|(t)=|\langle\nabla f, \dot{\gamma}\rangle|(t) \leq|\nabla f|(\gamma(t)) \leq \sqrt{f(\gamma(t))}=\sqrt{h(t)} .
$$

By integrating the above inequality, we get $|\sqrt{h(d(x))}-\sqrt{h(0)}| \leq \frac{1}{2} d(x)$. Thus $|\nabla f|(x) \leq \frac{1}{2} d(x)+\sqrt{f(p)}$.

Finally, we consider that the soliton is expanding. Similarly we only need to show the normalized case. So $R \geq-\frac{C}{2}$ and $R+|\nabla f|^{2}+f=0$. We obtain $-f+\frac{C}{2} \geq|\nabla f|^{2}$. Let $h(t)=-f(\gamma(t))+\frac{C}{2}$. Thus

$$
\left|h^{\prime}\right|(t)=|\langle\nabla f, \dot{\gamma}\rangle|(t) \leq|\nabla f|(\gamma(t)) \leq \sqrt{h(t)}
$$

By integrating the above inequality, we get $|\sqrt{h(d(x))}-\sqrt{h(0)}| \leq \frac{1}{2} d(x)$. Thus $|\nabla f|(x) \leq \frac{1}{2} d(x)+\sqrt{-f(p)+\frac{C}{2}}$.

Therefore we have proved (i).

The conclusion (ii) follows from (i) immediately.

Step 3. Since the gradient field $\nabla f$ grows at most linearly, it must be integrable. Thus we have proved Theorem 1.3.

\section{ACKNowledgement}

I would like to thank my advisor, Professor X.P. Zhu, for many helpful suggestions and discussions.

\section{REFERENCES}

1. S. Bando, On three-dimensional compact Kähler manifolds of nonnegative bisectional curvature, J. Diff. Geom. 19 (1984), 283-297. MR755227(86i:53042)

2. B. Chow, P. Lu, and L. Ni, Hamilton's Ricci flow, Graduate Studies in Mathematics, Amer. Math. Soc., Providence, RI, 2006.

3. B. Chow, and D. Knopf, The Ricci Flow: An Introduction, Mathematical Surveys and Monographs, vol. 110, Amer. Math. Soc., Providence, RI, 2004. MR2061425 (2005e:53101)

4. R. Schoen and S. T. Yau, Lectures on differential geometry, Conference Proceedings and Lecture Notes in Geometry and Topology, 1, International Press Publication, 1994. MR 1333601 (97d:53001)

Department of Mathematics, Sun Yat-sen University, Guangzhou, People's Republic OF CHINA 510275

E-mail address: juhoncheung@sina.com 